\title{
Major depression is common in people over the age of 50 , particularly in those at socioeconomic disadvantage, or with poor physical health and previous depressive symptoms
}

Mojtabai R, Olfson M. Major depression in community-dwelling middle-aged and older adults: prevalence and 2- and 4-year follow-up symptoms. Psychol Med 2004;34:623-34.

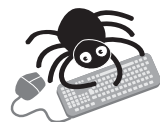

This article

contains extra text on the EBMH website

Do demographic, socioeconomic, and clinical factors increase major depression in middle aged and older adults?

\section{METHODS}

ए

Design: Prospective longitudinal study.

Setting: General population, USA; 1996-2000

of Population: 9747 community dwelling people taking part in the Health and Retirement study (aged $>50$ years in 1996).

Exclusions: not having completed a structured interview for major depression at enrolment.

(1) Assessment: People with major depression were identified using the short form of the Composite International Diagnostic Interview. Diagnosis of depression required five or more depressive symptoms in the same two week period during the year preceding the interview. The short form of the Center for Epidemiological Studies Depression scale was used to assess the severity of depressive symptoms occurring in the week preceding the interview. People were defined as having persistent significant depressive symptoms if they had significant depressive symptoms at all assessments. Most interviews were conducted by telephone. Twelve month prevalence and variables associated with major depression and persistence were assessed using multivariate logistic regression and contingency tables.

四央

Outcomes: Major depression.

Follow up period: Four years.

\section{MAIN RESULTS}

The 12 month prevalence of major depression was $6.6 \%$ in people over the age of 50 (95\% CI 6.1 to 7.1$)$. Prevalence in different subgroups is presented in the table (see http://www.ebmentalhealth. com/supplemental for table). In general, major depression was more common in: younger people; people who had separated from partners; people who were less educated and of lower socioeconomic status. Persistent significant depressive symptoms were associated with age, race, marital status, current employment, income, assets, physical illness, severity of major depression, and psychiatric treatment at baseline (data not shown).

\section{CONCLUSIONS}

Major depression is common in older people, particularly in those at socioeconomic disadvantage, or with poor physical health and previous symptoms of depression. Bronx, NY 10463, USA; rm322@columbia.edu

Sources of funding: National Institute of Mental Health, USA.

\section{NOTES}

This report uses data from third to fifth rounds of the Health and Retirement Study, a longitudinal study of community dwelling adults in the USA. African-Americans, Hispanics, and Florida residents were overrepresented.

\section{Commentary}

7 his article reports results of an epidemiological study (US Health and Retirement Study (HRS)) regarding the prevalence of major depression and persistence of symptoms assessed in a nationally representative community sample of middle aged and older adults in 1996, 1998, and 2000. This ambitious longitudinal survey is relevant because the results suggest that the persistence of depressive symptoms seems to increase with age although, surprisingly, the prevalence of major depression seems to decline with age. Moreover, the results indicate that the persistence of significant depressive symptoms is associated with socioeconomic disadvantage, physical disease (particularly recent stroke and heart attack), and a certain symptom triad at baseline (anhedonia, feelings of worthlessness, thoughts of death). Thus, identification of these patients is most important because they may need intensive treatment and careful longitudinal monitoring. Unfortunately, the disease is frequently not recognised in these age groups in primary care. ${ }^{1}$

The report is impressive because the representative sample was large and the authors applied the Composite International Diagnostic Interview ${ }^{2}$ and the short form of the Center for Epidemiological Studies Depression Scale ${ }^{3}$ as suitable measures of major depressive episodes in the elderly. ${ }^{4}$ If we take the results seriously, they would suggest that it is necessary to concentrate more efforts on those middle aged and older people suffering from depression who, due to socioeconomic disadvantages, have difficulty obtaining an effective treatment for depression.

The generalisability of this study is limited to middle aged and older adults in the general population of the United States. Future research should also address institutionalised populations because the prevalence of the disease may be higher and the depressive disorder may be more severe in terms of symptoms and duration. In future research it would be advantageous to avoid some methodological issues of the HRS through more detailed analysis of the treatment, determining physical illness using clinician reported information and focusing on the subgroup of elderly patients (minimum age, 70 years) in order to obtain more reliable information about factors associated with the persistence of depression in this frequently neglected age group suffering from major depression.

Verena Henkel, MD, Roland Mergl, PhD, Hans-Jürgen Möller, MD

Department of Psychiatry, Ludwig-Maximilians-Universität Munich, Munich, Bavaria, Germany

1 Henkel V, Mergl R, Kohnen R, et al. Use of brief depression screening tools in primary care: consideration of heterogeneity in performance in different patient groups. Gen Hosp Psychiatry 2004;26:190-8.

2 Kessler RC, Andrews G, Mroczek D, et al. The World Health Organization Composite International Diagnostic Interview Short-Form (CIDI-SF). Int J Methods Psychiatr Res 1998;7:171-85.

3 Radloff LS. The CES-D scale: the self-reported depression scale for research in the general population. Appl Psychol Management 1977;1:385-401.

4 Turvey CL, Wallace RB, Herzog R. A revised CES-D measure of depressive symptoms and a DSM-based measure of major depressive episodes in the elderly. Int Psychogeriatr 1999;11:139-48. 